ISSN: $1135-9250$

EDUTEC EDUTEC. Revista Electrónica de Tecnología Educativa.

Número 61/ Noviembre 2017

\title{
CARACTERIZACIÓN DEL PERFIL DE LOS ESTUDIANTES DE SECUNDARIAS EN EL ACCESO Y USO DE INTERNET A PARTIR DE LAS TIC.
}

\section{CHARACTERIZATION OF THE PROFILE OF HIGH SCHOOL STUDENTS IN INTERNET ACCESS AND USE FROM THE ICT.}

\author{
Carlos René Contreras Cázarez; renecazarez@gmail.com \\ Reyna Campa Álvarez; reyna.campaal@gmail.com \\ Universidad de Sonora \\ México
}

\section{RESUMEN}

En la última década han proliferado los estudios de acceso y uso de Internet en adolescentes. La mayoría de estas investigaciones describen y analizan de manera útil los hábitos de consumo y preferencias de uso. La finalidad del presente artículo es analizar y evaluar la validez y confiabilidad de un modelo teórico hipotético propuesto para el estudio de acceso y uso de Internet en los estudiantes de secundarias. El estudio se deriva de un proyecto de investigación más amplio realizado en Hermosillo, Sonora, México. La muestra la conforman 225 estudiantes de escuelas secundarias. El análisis factorial exploratorio sugirió la existencia de dos o más factores que influyen en el acceso y uso de Internet para la caracterización del perfil de los estudiantes. Los resultados fueron coherentes con la teoría al establecer que los jóvenes prefieren mayoritariamente los procesos de socialización en línea como parte del acceso y uso de Internet.

PALABRAS CLAVE: Socialización en línea; TIC; comunicación; jóvenes de secundaria; habilidades digitales; acceso y uso de Internet.

\section{ABSTRACT}

In the last decade, Internet studies of access and use in adolescents have proliferated. Most of these investigations describe and analyze in a useful way the habits of consumption and preferences of use. The purpose of this article is to analyze and evaluate the validity and reliability of a hypothetical theoretical model proposed for the Internet study of access and use in high school students. The study is derived from a larger research project conducted in Hermosillo, Sonora, Mexico at Public High School. The sample is made up of 225 at publics schools students. The 
exploratory factor analysis suggested the existence of two or more factors that influence the access and use of the Internet for the characterization of the profile of the students. The results were consistent with the theory by stating that young people mostly prefer online socialization processes as part of Internet access and use.

KEYWORDS: Online socialization; ICT; communication; high school students; digital skills; Internet access and use.

\section{INTRODUCCIÓN}

Uno de los principales retos que enfrenta la sociedad actual son las constantes transformaciones del desarrollo de las Tecnologías de la Información y la Comunicación (TIC). Internet se convierte, en este sentido en una herramienta que posibilita, afecta y transforma las diferentes esferas de la vida social y profesional. Por otra parte, es evidente que las sociedades contemporáneas están adoptando los cambios e innovaciones que derivan de un mundo más conectado por el uso de estas herramientas. La conectividad en este sentido involucra desde luego, el contexto educativo, dónde los actores principales, los estudiantes participan de manera activa en el proceso de aprendizaje. Hoy en día es muy sencillo acceder a los diferentes contenidos informativos de interés general. Así el uso de Internet ha enriquecido los diversos escenarios en los que se desarrollan y practican las relaciones interpersonales y particularmente de comunicación y educación.

Los estudios que se han pronunciado en los últimos años para describir y explicar las prácticas comunicativas de las generaciones interactivas o nativos digitales muestran, por un lado, los usos de Internet más frecuentes de los adolescentes, en los que se evidencian hábitos de consumo y los procesos socialización. Las tecnologías les facilitan a estos jóvenes el conocimiento sobre el mundo, viniendo a sustituir otras formas tradicionales de aprendizaje y acceso a la información. Por otro lado, internet y las redes sociales se han convertido para estos nativos digitales en los escenarios en los que gestionan todas sus relaciones vitales (de amistad, de ocio...), lo que no está exento de conflictos y riesgos (Hernández, F., Alcoceba H., 2015).

En este sentido, los procesos de socialización en línea para los jóvenes, se percibe como el elemento integrador para la construcción de la identidad personal y grupal de los adolescentes (Hernández, et. al. 2015). De igual forma, se expresa en otras investigaciones que el uso de Internet y las redes sociales son importantes tanto para el desarrollo emocional y social de los jóvenes. Así el mundo virtual funciona como su patio de recreo, para los problemas del desarrollo, tales como la auto-identidad y la sexualidad (Gross, 2004). Las redes sociales ofrecen intimidad a los jóvenes, en donde pueden crecer y madurar en espacios privados (Bartolomé, 2014), aunque en ocasiones se corre el riesgo de no seguir la orientación adecuada.

Las aportaciones sobre la socialización en línea de los adolescentes también han sido de suma importancia en las investigaciones de hábitos de consumo de los jóvenes. En varios estudios 
trasnacionales, particularmente en España, en relación al uso de las TIC, se encontró que una de las actividades más habituales en Internet, según el estudio presentado por Cogo, ElHajji y Huertas (2012) son las relaciones sociales con pares, aunque el porcentaje es superior entre los usuarios nacidos en España, (91,4\% frente 79,8\% los migrantes). En otras latitudes, en México, la situación revela que los servicios de Internet que utilizan las y los jóvenes de secundaria son muy variados. Los más usados son las redes sociales con un $83 \%$ de preferencias y el uso de mensajes instantáneos (vía Twitter, WhatsApp, Messenger o cualquier aplicación de mensajería multiplataforma que le permite enviar y recibir mensajes sin pagar) es lo que prefiere al menos la mitad de los jóvenes bajo estudio (Contreras, León, y Moreno, 2014).

En otras investigaciones, se enuncia que las redes sociales no pretenden profundizar en las relaciones sino establecerlas y mantenerlas, con comentarios, fotos, respuestas a otros comentarios, compartiendo enlaces de interés, etc., siempre con intervenciones breves, única forma que permite la propia estructura de la red social (Reyero, García, Hernández y Ovide, 2011).

En Europa, las aportaciones sobre hábitos de consumo y socialización son muy variados. Cada año se reportan informes de autores como Bringué, Sádaba y Tolsá (2011), Sánchez Burón y Álvaro Martín (2011), Fumero y Espíritusanto (2012 en Ruíz Corbella, M., Juana O., (2013), que evidencian la utilización de las redes sociales como medio de interacción principal en la población analizada. Otros estudios de compañías telefónicas, como la Fundación Orange (2012), Fundación Telefónica (2012) y Fundación Pfizer (2009), revelan que 9 de cada 10 usuarios utilizan las redes sociales para estar en contacto con amigos con los que se ven con frecuencia. Los diversos estudios presentan semejanzas tanto en los sectores de la población que es estudiada, así como los rangos de edades. Sin embargo, coinciden que los procesos de socialización e interacción de los adolescentes son hoy en día un medio imperante para la construcción de la identidad. Otras investigaciones analizan las repercusiones del uso de las TIC en la socialización de los jóvenes, en tanto que la socialización está mediada por el conjunto de sus relaciones sociales y el uso de Internet, Bernete, F., (2010).

La situación en México no dista de lo que ocurre en otros países al reflejar que la red social preferida es Facebook, con el $98 \%$ de la muestra de jóvenes conectados, seguida por Twitter con el 69\%, (IAB México y Millward Brown, Televisa, 2012). Lo mismo ocurre en otras regiones de la república mexicana, dónde un dato porcentual considerable del $84 \%$ de participación de jóvenes afirmó utilizar redes sociales (León, D., Caudillo, R., Contreras, C., Moreno, C., 2014). Dos años más tarde, según el estudio presentado por los mismos autores, el dato porcentual se había incrementado al 92.3\% de participación de estudiantes en el uso de redes (León, D., et. al., 2015).

En cuanto al equipamiento y conectividad a Internet también se han realizado algunas investigaciones relevantes en el caso de Latinoamérica. Para el año del 2011, el sistema de estadísticas de la Comisión Económica para América Latina (CEPAL) y el Caribe de la ONU evidenció que el promedio de hogares con Internet alcanzaba el 29.7\%. Uruguay, Argentina y Chile tienen proporciones de poco más del 40\%, mientras que la proporción en México es del $30.7 \%$ al 2013. En el mismo contexto, particularmente en México, se evidenció cifras porcentuales de suma importancia en cuanto al equipamiento de dispositivos tecnológicos en el hogar y 
conectividad a Internet, como es el caso del estudio presentado por León, D., et. al., $(2014,2015)$ en donde se revela que el $91 \%$ de los estudiantes opinó tener computadora en casa y el $85 \%$ manifestó tener celular; de la misma forma se dio cuenta del acceso a Internet en el hogar de los estudiantes al afirmar que el 83\% dijo tener conexión a esta herramienta.

Por ello los estudios de acceso y uso han sido cruciales en las recientes investigaciones. La literatura relevante ha señalado que numerosos factores influyen tanto en la introducción y la aceptación de las TIC en los entornos educativos: la cognición personal, profesional y la eficacia, la falta de conocimientos, habilidades y la dinámica socioeconómica desfavorable (Fanni et al., 2010; Van Zyl y Rega, 2011). En nivel de acceso se ha convertido en un indicador de desarrollo de los países, no solo en el ámbito económico, sino también en el desarrollo de la sociedad del conocimiento. Por su parte, el Informe Mundial sobre el Estado de la Banda Ancha ONU (2014) presentado en la UNESCO (2017), expresa que más del 50\% de la población mundial tendrá acceso a Internet dentro de los próximos tres años, dado que la banda ancha móvil a través de teléfonos inteligentes y tabletas es la tecnología que más rápidamente crece en la historia humana.

Al desarrollo y estudio de las habilidades digitales que deben poseer todos los actores participantes en el proceso de enseñanza-aprendizaje, principalmente en educación básica, diferentes autores han realizado aportaciones para analizar y medir su efecto. Quintana, Cortada y Riera (2012) identificaron que los estudiantes de primaria y secundaria que tienen un mayor contacto con las TIC saben cómo usar la tecnología, pero no presentaron diferencias para la búsqueda de información en internet. Además, todos estos estudiantes muestran un mejor conocimiento y control de la computadora, así como mejores habilidades digitales.

Por otra parte, el estudio realizado por la Organización para la Cooperación y el Desarrollo Económico (OCDE u OECD por sus siglas en inglés) del año 2009, Ilamado PISA ERA, midió las habilidades digitales de los estudiantes de 16 países, además de tres países invitados (OECD, 2011). En dicho estudio se explicó que para recolectar información se requieren habilidades de "lectura rápida" (skimming and scanning) a través de grandes volúmenes de material que deben evaluarse en su credibilidad de manera inmediata. Se planteó que el pensamiento crítico de los estudiantes es más relevante para la alfabetización digital de lo que lo fue para la alfabetización de lectura impresa (Halpern, 2008; Shetzer \& Warschauer, 2000).

Como se ha evidenciado en estudios previos, existen algunas investigaciones que involucran las habilidades digitales con el acceso y uso de Internet de los adolescentes; al mismo tiempo que existen otras aportaciones que demuestran el uso generalizado de las redes sociales y los procesos de socialización como parte del uso de esta herramienta. Por consiguiente, el objetivo principal de este artículo es analizar y evaluar la validez y confiabilidad de un modelo estructural propuesto para el estudio que involucra a las variables de socialización en línea, habilidades digitales, acceso y uso de Internet en jóvenes de secundaria.

\subsection{Acceso y Uso de Internet.}

La literatura señala que el consumo debe entenderse dentro del proceso social como parte de las necesidades de la gente de relacionarse entre sí, así como parte de la necesidad de mediadores 
materiales en esas relaciones, Douglas e Isherwood (1996). La aportación que hacen los autores para establecer el vínculo de la apropiación y el uso de los bienes y servicios, refiere, en primer lugar, a la tecnológica; en segundo lugar a la de interacción social (acompañada por el gasto o consumo en diversiones, viajes, reuniones, invitaciones, fiestas, relaciones públicas, la asociación a un club, etc.); y por último, la de la relación informativa del consumidor (el acceso a servicios educativos, de asesoría, agentes de bolsa, servicios bancarios, etc.) o mediante el gasto en las relaciones sociales (contactos vitales para la obtención y conservación de un alto potencial de ganancia).

Para algunos autores, el uso, entendido en un sentido amplio, engloba todo lo relacionado con el acceso y a la utilidad que los jóvenes hacen de los medios de comunicación. Bringué; Sábada y Rodríguez, señalan que cuestiones como el equipamiento tecnológico en los hogares, el lugar de acceso a los diversos medios, el momento del día en el que los utilizan o la cantidad de tiempo que les dedican; son algunos de los indicadores principales para medir las pautas de consumo de Internet (Bringué; Sábada y Rodríguez., 2009). Para Muñoz y Antón, el acceso y uso de contenidos digitales ha de ser entendido en el contexto global de la sociedad de la información, donde Internet tiene una presencia constante y ubicua, y el ocio, el aprendizaje en la educación y lo laboral se relacionan estrechamente. Los usos de estos contenidos están sustentados y mediados por las TIC y por los valores de esta era de la información, postindustrial y de consumo donde resulta difícil desvincular lo digital de Internet (Muñoz, L., \& Antón, M., 2017). En este mismo orden de ideas, se asume que uno de los principales indicadores como parte de los servicios y contenidos de Internet son las redes sociales. Las múltiples plataformas y aplicaciones de socialización se están convirtiendo día a día en el principal mecanismo de relación y socialización entre los consumidores. De hecho, el uso de redes sociales ha crecido de forma notable según los estudios analizados, situándose como una de las principales vías de acceso para el consumo de contenidos. En este sentido, Bernal \& Angulo (2012) afirman que el uso y acceso de las redes sociales ha llegado a ser un nuevo entorno de socialización para los jóvenes, espacio para la construcción de la identidad social con sus iguales en ocasiones sin ningún control o supervisión parental.

\subsection{Habilidades digitales}

Cuando se habla de habilidades digitales, se alude inevitablemente a la incorporación de estas competencias a las TIC. Sin lugar a dudas, las habilidades digitales en la educación contribuyen a que los estudiantes desarrollen la competencia digital que implica ser una persona autónoma, eficaz, responsable, crítica y reflexiva al seleccionar, tratar y utilizar la información y sus fuentes, además de sus herramientas tecnológicas (Area, 2009). Por su parte, Revuelta (2011) explica que "la competencia digital se sustenta en las competencias básicas en materia de TIC: el uso de ordenadores para obtener, evaluar, almacenar, producir, presentar e intercambiar información y comunicarse y participar en redes de colaboración a través de internet" (p. 3). En otra definición, la autora manifiesta que la competencia digital implica el uso seguro y crítico de las tecnologías de la sociedad de la información (Revuelta, 2011: 3). Así, la capacidad requerida para manejo crítico de la información cobra una importancia vital en los entornos virtuales y las personas deben contar con las habilidades necesarias para acceder a bases de datos en Internet. 
Paralelamente, en otra definición, Lozano (2010), expresa que la sociedad del conocimiento promueve las habilidades de la información, es decir, la capacidad de una persona para reconocer sus necesidades de información, localizarla, recopilarla, evaluarla y utilizarla de manera efectiva.

Según el informe presentado por la OECD de habilidades y competencias digitales para el siglo XXI, advierte que la explosión informativa desencadenada por las TIC requiere nuevas habilidades de acceso, evaluación y organización de la información en entornos digitales. Del mismo modo, se argumenta que en aquellas sociedades donde el conocimiento tiene un papel central, se requiere de la habilidad para modelarla, transformarla y generar nuevo conocimiento o para usarlo como fuente de nuevas ideas (OECD, 2010).

En este mismo orden, las habilidades digitales o competencias digitales de la generación interactiva en el siglo XXI, exige a las nuevas generaciones del conocimiento, habilidades de búsqueda, selección, evaluación y organización de la información en entornos digitales. Las típicas habilidades pertenecientes a esta dimensión son habilidades de investigación y resolución de problemas, que conllevan en algún punto definición, búsqueda, evaluación, selección, organización, análisis e interpretación de la información (OCDE, 2010). Siguiendo en la misma línea, se señala que la gran masa de información disponible en Internet y la proliferación de bases de datos exigen encontrar y organizar rápidamente información y desarrollar cierta habilidad de discriminación de la información. Así pues, la competencia digital entraña el uso seguro y crítico de las tecnologías de la sociedad de información para el trabajo, el ocio y la comunicación. Por su parte, la Reforma Integral de Educación en México RIEB (2011), explica que parte de las competencias que el estudiante debe adquirir durante su formación es la búsqueda, selección, análisis y evaluación proveniente de diversas fuentes; además de aprovechar los recursos tecnológicos a su alcance, coma medios para comunicarse, obtener información y construir conocimiento.

\subsection{Socialización en línea}

Cuando se habla de socialización, es referirse desde luego a un término bastante amplio y complejo. En la literatura académica, diferentes autores definen la socialización en términos generales como el proceso en el cual los individuos incorporan normas, roles, valores, actitudes y creencias, a partir del contexto socio-histórico en el que se encuentran insertos a través de diversos agentes de socialización tales como los medios de comunicación, la familia, los grupos de pares y las instituciones educativas, religiosas y recreacionales, entre otras (Arnett, 1995; Maccoby, 2007; Grusec y Hastings, 2007). De igual forma, Colás, González \& De Pablos (2013) señalan que algunas aportaciones recientes muestran que la comunicación social en línea de jóvenes está influida por la percepción de su identidad y autoestima, así como la compensación social y entorno social.

Desde las disciplinas de las Ciencias Sociales, las contribuciones al término de socialización han sido innumerables. La sociología, ha contribuido al definir a la socialización como un proceso de conformación y organización de los grupos sociales en el cual se ven implicados factores políticos, económicos, simbólicos y culturales, St. Martin (2007). En otra definición del mismo campo, 
Berger y Luckmann (1968) proponen una definición bastante amplia del concepto y describen que la socialización primaria corresponde a la introducción del individuo en la sociedad, es decir, a la internalización por parte del sujeto. En una definición más puntual, los autores refieren que "la socialización primaria es la primera por la que el individuo atraviesa en la niñez; por medio de ella se convierte en miembro de la sociedad [...]. Se advierte, además, que la socialización primaria suele ser la más importante para el individuo y que la estructura básica de toda socialización secundaria debe semejarse a la primaria" (Berger y Luckmann, 1968:168). De esta forma, la interacción se convierte en un aspecto importante de la socialización y puede ser una medida de la socialización.

En un sentido más amplio, cuando se habla de interacción, el concepto frecuentemente está relacionado con las tecnologías de la información y la comunicación, en especial con Internet. Sin embargo, es posible identificar que los medios centrales de interacción social en los adolescentes son las redes sociales Contreras, C., (2017). Así pues, las redes sociales, se perfilan como el espacio idóneo no solo para los estudiantes y sus pares, sino también para la interacción con sus padres, profesores, personas conocidas y desconocidas.

Por tanto, es posible afirmar en palabras de Bernal y Rivas (2012) que las relaciones entre padres e hijos suponen aspectos afectivos y cognitivos: apoyo, cariño, disponibilidad para con los hijos. Todos estos elementos tienen que ver con las expectativas, el control, el establecimiento de límites y de orientación de los padres para con sus hijos. En este sentido, estos elementos del contexto familiar contribuyen y son predictivos de adaptación social, desarrollo de competencias sociales y conductas prosociales (Hillaker B., Brophy-Herb, H., Villarruel, F. y Haas, B., 2008), en Ibídem, (2012). Por otra parte, es importante mencionar que es por medio de las interacciones familiares donde se reflejan y transmiten todo tipo de prácticas que se encuentran presentes en un contexto socio cultural.

El proceso de socialización sugiere la interacción de diferentes agentes individuales, grupales y sociales. Dentro de la clasificación de los agentes involucrados en el proceso de socialización se encuentran la familia, los grupos de amigos o pares, la escuela, así como los medios de comunicación. Particularmente este último, refiere al acceso y uso de las TIC, donde de acuerdo con Arnett (1995) las sociedades varían en relación a la cantidad y el acceso a los medios de comunicación que poseen sus miembros. De este modo, "el que está aislado y marginado ya no es, necesariamente, el que no tiene gente a su alrededor, sino el que está desconectado [...] En términos de visibilidad social, lo que no puede ser visto en los medios o subido a la red "no existe". (Winocur, 2009).

Por su parte, los autores Ballesta, Lozano y Cerezo, afirman que la mayoría de los alumnos utilizan las redes sociales principalmente para divertirse y ponerse en contacto con sus amigos, también para conocer otros e intercambiar mensajes e imágenes, una actividad que realizan desde hace tiempo y que reconocen no quitar tiempo a otras actividades (Ballesta, Lozano \& Cerezo, 2014). 


\section{MÉTODO.}

\subsection{Muestra.}

La muestra la constituyen 225 estudiantes de secundarías públicas mexicanas en la localidad de Hermosillo, Sonora, México. Cabe señalar que los estudiantes además de responder a los ítems del instrumento, se recabó información de otras variables como el sexo, edad, y grado escolar. Lo cual permitió conocer más sobre la selección de la muestra.

\subsection{Instrumento.}

El instrumento forma parte de un estudio macro de Internet y relaciones interactivas en jóvenes de secundarias públicas mexicanas, León, D. Caudillo, R., Contreras, C., Moreno, C., (2014), que mide las variables de acceso y uso de Internet, habilidades digitales, socialización, confianza, riesgos y dependencia de Internet. Para efecto de la validez y confiabilidad del modelo estructural propuesto para este estudio, se tomó los ítems de las variables correspondientes, únicamente de acceso y uso, socialización y habilidades digitales.

El factor de acceso y uso está conformado por 37 ítems, los cuales refiere a los contenidos de navegación, servicios de navegación, así como el tiempo de uso. En tanto, el factor de socialización en línea, el cual está constituido por 24 ítems, señala los procesos de interacción entre pares -compañeros de clase, familiares, personas conocidas y desconocidas. Por último, el factor de habilidades digitales está conformado por 33 ítems, el cual refiere a la búsqueda, selección, referenciar la información, así como uso de programas y creadores de contenidos.

\subsection{Procedimiento}

Los estudiantes fueron encuestados en la institución educativa con previo aviso y permiso de la dirección administrativa. Con el apoyo del profesor de aula se aplicó el cuestionario a los participantes de los tres grados de secundaria. La duración de aplicación fue aproximadamente entre 35-40 minutos. Para responder a los ítems, el estudiante debía señalar a cada una de las afirmaciones mediante una escala tipo Likert con cinco alternativas de respuesta: $(0=$ nunca, $1=$ rara vez, 2 = algunas veces, 3 = casi siempre y $4=$ siempre).

\subsection{Análisis de los datos.}

Se realizó un Análisis Factorial Exploratorio (AFE) para conocer el número de factores que subyacen al modelo hipotético propuesto. Para obtener los datos estadísticos fue necesario agrupar los datos en escalas, obteniendo de este modo: medias, desviaciones estándar, valores mínimos y máximos de cada uno de los factores, así como el alfa de Cronbach que indica la confiabilidad de la escala. De esta forma, los valores y las parcelas se utilizaron para determinar el número de factores a extraer en el modelo hipotético para el estudio en cuestión. Dado el tamaño de la muestra, y las alfas de las escalas se prosiguió a la extracción de los factores.

Por último, se realizó un Análisis Factorial Confirmatorio (AFC) en el programa EQS versión 6.1, donde se analizó la coherencia y validación de los factores determinando la relación y la 
direccionalidad de los mismos. Para tal estudio, los índices de ajuste requeridos fueron el CFI (Bentler, 1990), el de Bentler-Bonett de Ajuste No Normado (NNFI, en sus siglas en inglés, Bentler y Bonett, 1980); La Raíz Cuadrada del Cuadrado Medio del Error de Aproximación (RMSEA, en sus siglas en inglés; Steiger, 1990); de la misma manera, la teoría estadística, establece, que el índice de Ajuste Comparativo (CFI, en sus siglas en inglés para considerarse aceptable debía ser > .90; Bentler, 1992). El AFC arrojó para el estudio en cuestión que los valores fueron mayores a este indicador.

\section{RESULTADOS.}

Para la validez del instrumento, se calcularon las alfas de Cronbach de las escalas que integran el instrumento. Como se puede observar el alfa total del instrumento fue de .86, lo cual indica fiabilidad del mismo. De la misma manera, las alfas obtenidas en cada una de las escalas fueron mayor de .60 por lo cual se consideran aceptables y fiables (ver Tabla 1)

\begin{tabular}{lcccccc}
\hline Escalas & $\mathrm{N}$ & Mínimo & Máximo & Media & D.E & Alfa \\
\hline & & & & & & .86 \\
& & & & & & \\
\hline Socialización en línea & 225 & 0 & 4 & 4.38 & .868 & .90 \\
\hline Habilidades digitales & 225 & 0 & 4 & 3.25 & .643 & .68 \\
\hline Acceso y Uso de Internet & 225 & 0 & 4 & 4.22 & .812 & .88 \\
& & & & & & \\
\hline
\end{tabular}

Tabla 1. Alfas de Cronbach de las escalas (Elaboración propia)

Los datos descriptivos, por su parte, muestra medias bajas en lo que refiere a las habilidades digitales de los estudiantes en el acceso y uso de Internet con 3.25, seguido del acceso y uso con una media 4.22, mientras que la socialización en línea mostró una media de 4.38.

La tabla 2 muestra la prueba de correlación de Pearson. Se halló una relación positiva entre el acceso y uso de Internet con las habilidades digitales de los estudiantes de secundaria; lo que significa, que la generación interactiva de jóvenes a mayor acceso y tiempo de uso de esta herramienta, mayor conocimiento y dominio presentan en sus habilidades digitales. De igual forma, se encontró una correlación positiva de la socialización en línea con el acceso y uso de Internet, lo que indica, que los jóvenes acceden y usan el Internet principalmente para fines de interacción y socialización, en el que comparten mayor parte de su tiempo interactuando con sus iguales, padres, personas conocidas y desconocidas. Por otra parte, el estudio encontró una correlación negativa de la socialización en línea con las habilidades digitales de los estudiantes. 


\begin{tabular}{cccc}
\hline & $\begin{array}{c}\text { Habilidades } \\
\text { digitales }\end{array}$ & $\begin{array}{c}\text { Socialización } \\
\text { en línea }\end{array}$ & Acceso y Uso de Internet \\
\hline Habilidades digitales & 1 & & \\
\hline Socialización en línea & $-0,238^{*}$ & 1 & 1 \\
\hline $\begin{array}{c}\text { Acceso y Uso de } \\
\text { Internet. }\end{array}$ & $0,227^{*}$ & $0,122^{*}$ & \\
\hline
\end{tabular}

Tabla 2. Correlaciones (Pearson) entre las variables de estudio

* La correlación es significante al nivel 0,05 (bilateral).

La Figura 1 muestra la relación del modelo teórico hipotético propuesto para este estudio, en el que las variables latentes se representan mediante círculos, mientras que las variables observadas en rectángulos. En tanto, la relación entre cada uno de los factores del modelo aparece mediante la representación de flechas.

El modelo de ecuaciones estructurales muestra que los factores de socialización en línea, habilidades digitales, así como el acceso y uso de Internet se formaron de manera congruente con sus indicadores. Así, el modelo de medición no sólo obtuvo indicadores de validez de constructo de las variables latentes, sino que, además, señala la validez convergente.

El factor de acceso y uso de Internet se formó con la variable contenidos de navegación (peso factorial=.73), la variable tiempo de uso (peso factorial $=.71$ ) y servicios de navegación (peso factorial= .83). De la misma forma, el factor de socialización en línea se formó congruentemente con las variables interacción con los pares (peso factorial=.72), la variable interacción con los padres (peso factorial $=.74$ ), interacción con personas desconocidas (peso factorial= .64) e interacción con personas conocidas (peso factorial $=.76$ ). Finalmente, el factor de habilidades digitales se formó de manera significativa con las variables búsqueda de información (peso factorial= .68), selección de la información (peso factorial $=.72$ ), referenciar la información (peso factorial $=.63$ ), y uso de software y creadores de contenido (peso factorial $=.67$ ).

El modelo de ecuaciones estructurales sobre la caracterización del perfil de los estudiantes de secundarias en el acceso y uso de Internet mostró que el factor de socialización en línea tuvo un efecto directo y fuerte con el acceso y uso de Internet (coeficiente estructural = .92). En una interpretación del modelo, los jóvenes cuando acceden a esta herramienta lo hacen principalmente con la finalidad de interactuar y socializar con sus iguales. Así, el acceso a los diferentes medios y dispositivos tecnológicos facilita la interacción de los jóvenes en sus procesos de socialización. Cómo se ha señalado en líneas anteriores, el uso en un sentido amplio engloba todo lo relacionado con el acceso y a la utilidad que los jóvenes hacen de los medios de comunicación. Cuestiones como el equipamiento tecnológico de los hogares, el lugar de acceso a los diversos medios, el momento del día en el que los utilizan o la cantidad de tiempo que les dedican (Bringué; Sábada y Rodríguez, 2009). 
De igual forma, el factor de habilidades digitales mostró un efecto directo positivo con el acceso y uso de Internet (coeficiente estructural $=.64$ ). En otra interpretación sobre el conocimiento o dominio de las habilidades digitales de los estudiantes de secundaria, los jóvenes presentan conocimientos básicos en cuanto a la utilización de recursos didácticos y prácticos para la elaboración de trabajos académicos. El estudio reveló que su relación con el acceso y uso de Internet fue directo y positivo por lo que los jóvenes utilizan esta herramienta para los trabajos escolares. Paralelamente, es posible asumir que los jóvenes realizan de manera simultánea actividades escolares como de ocio y entretenimiento.

Por otra parte, se ha evidenciado en otros estudios la importancia de las habilidades digitales de los estudiantes: la capacidad de reconocer sus necesidades de información, de localizarla, recopilarla, evaluarla y utilizarla de manera efectiva, así como el desarrollo de las técnicas adecuadas para hacerlo (Lozano, 2010). En el mismo sentido, Gutiérrez \& Tyner (2012) explican que algunas de las características de los nativos digitales o sociedad de la Información del siglo XXI son la digitalización de la información, así como la importancia de las redes sociales (...) De acuerdo con Revuelta (2011), se afirma que la competencia digital se vuelve una competencia básica en la actualidad, así como la capacidad del manejo crítico de la información y la habilidad de comunicación en los entornos virtuales.

De este modo, todos los indicadores de bondad de ajuste del modelo teórico hipotético propuesto para el estudio alcanzaron los valores requeridos para la congruencia y pertinencia del modelo. La $\mathrm{X}^{2}$ fue baja y no significativa al mostrar una $(p=.12)$, lo que indica que no existen diferencias entre el modelo teórico y el modelo propuesto. Los índices prácticos, Índice BentlerBonett de Ajuste No Normado (NNFI, por sus siglas en inglés), así como el Índice de Ajuste Comparativo (CFI) fueron mayores a .90 como se muestra en el modelo. El indicador de la Raíz Cuadrada del Cuadrado Medio del Error de Aproximación (RMSEA) tuvo un valor de .05 , y la $\mathrm{R}^{2}$ del modelo fue de .48, lo que muestra que el modelo en su conjunto explica el $48 \%$ de la varianza sobre la caracterización del perfil de los estudiantes en el acceso y uso de Internet. 


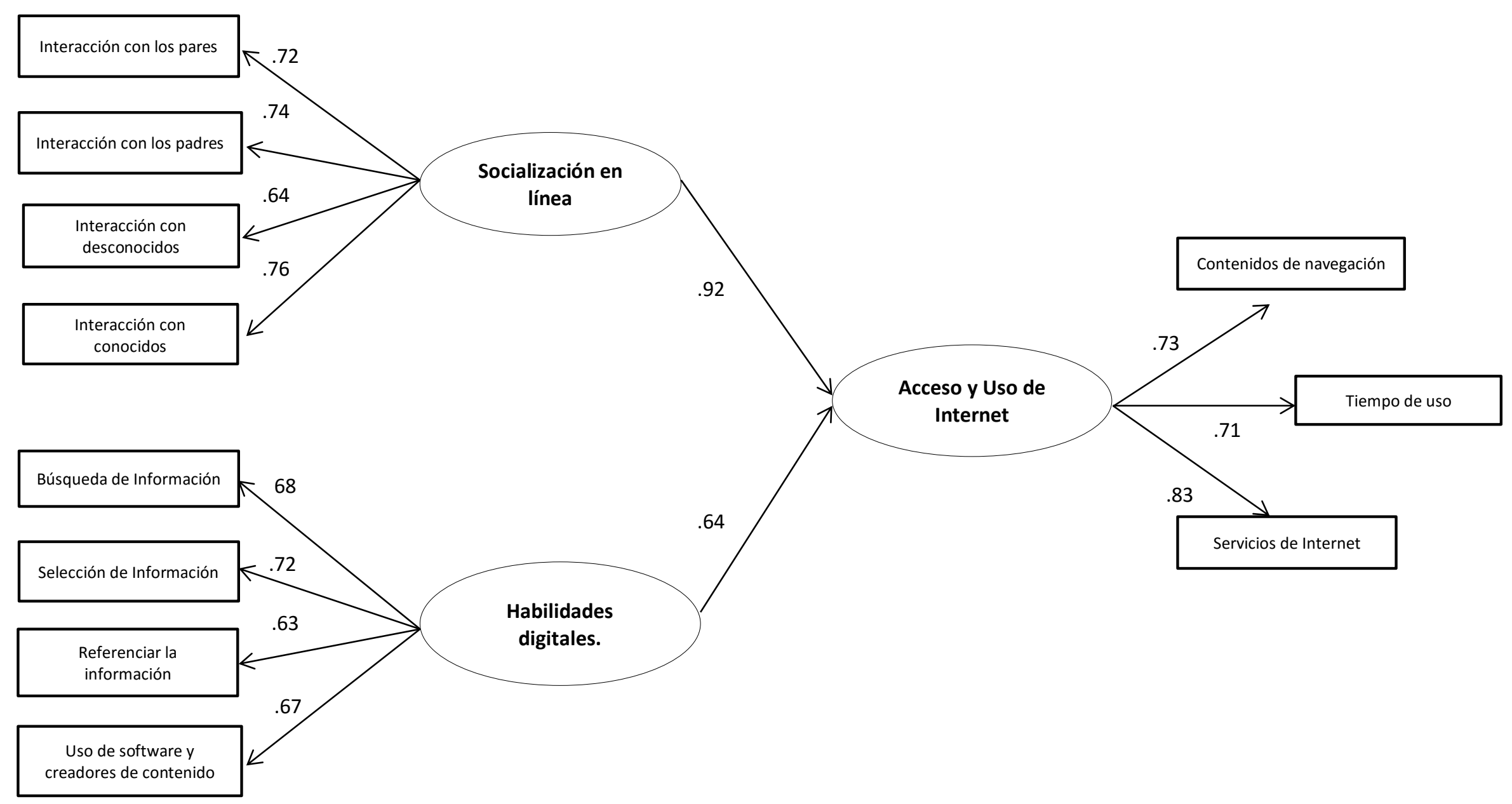

Figura 1. Modelo caracterización del perfil de estudiantes en el acceso y uso de Internet. $N=225, \mathrm{X}^{2}=33.68 ; \mathrm{GL}=32 ; \mathrm{P}=.12 ; \mathrm{BBNFI}:=.92 ; \mathrm{BBNNFI}:=.95 \mathrm{CFI}:=$ .95; RMSEA = .05; $\mathrm{R}^{2} .48$.

Nota: elaboración propia 


\section{DISCUSIÓN Y CONCLUSIONES.}

De acuerdo al modelo estructural propuesto para la caracterización del perfil de los estudiantes de secundarias públicas en Sonora, México, se pudo evidenciar que los adolescentes muestran una tendencia de acceso y uso de Internet para fines de interacción y socialización. El estudio reveló que los jóvenes utilizan primordialmente esta herramienta para compartir experiencias con sus compañeros de clase. En un contexto más mediado por los dispositivos móviles y conectividad a Internet, los procesos de socialización reconfiguran los marcos para la interacción social, acarreando nuevos retos para la convivencia y la relación de los jóvenes en sus procesos vivenciales (Huertas, 2012). Siguiendo con la idea de la autora, expresa que los procesos de socialización digital en los jóvenes latinoamericanos son similares a sus espacios de sociabilidad presencial: los adolescentes conforman sus relaciones en las redes virtuales con otros jóvenes con los que comparten también especio físico, en el barrio, colegio o instituto. (Huertas, 2012).

Por otra parte, el estudio de Internet y jóvenes de secundaria en México (2014) demostró que la mayoría de los estudiantes de secundaria prefieren socializar por medio de redes sociales la mayor parte de su tiempo, cuando no están en la escuela, los mismos adolescentes manifestaron estar siempre en contacto con sus compañeros de clase y amigos. El estudio en cuestión observó que las redes sociales tenían mayor afluencia por parte de los jóvenes de secundaria como parte de los servicios más utilizados con un $84 \%$ de participación. Según los encuestados, siempre están en contacto para estar informados de las tareas de clase o ponerse de acuerdo para salir de paseo (Léon, D., et. al., 2014). De igual forma, es posible afirmar que la aparición de las redes sociales ha venido a reconfigurar los ámbitos de actuación y de relación juveniles, tanto en sus dimensiones productivas (tareas escolares, formación, etc.), así como reproductivas como el ocio, las relaciones familiares y personales (Hernández, et. al., 2015) citado en Contreras, C., (2017).

Según el informe presentado por León, D., et al., (2014), además de las interacciones de los estudiantes con sus mismos compañeros de clase, los jóvenes expresaron en menor medida estar en contacto con personas desconocidas por medio de las redes sociales como Facebook, en donde permanentemente reciben solicitudes de amistad y en ocasiones fotografías comprometedoras de personas extrañas, incrementando los riesgos en sus procesos vivenciales y de socialización.

Es posible afirmar que el acceso y uso por parte de los estudiantes de secundaria en México es un hecho inexorable. La reconfiguración en sus procesos de interacción y de socialización de los adolescentes por medio de las redes sociales contribuyen a la identidad de los menores. Por consiguiente, se hace necesario que, desde el ámbito educativo, se eduque a ser crítico a los jóvenes con los posibles riesgos del uso y consumo de Internet, así como de las múltiples plataformas y redes sociales. De la misma manera que es importante informar a los padres o tutores responsables de la educación del menor sobre los riesgos a los que se exponen sus hijos en Internet sin supervisión. 
El modelo teórico propuesto para este estudio establece en términos generales que los jóvenes gustan de relacionarse con sus pares tanto de manera presencial como en el entorno digital, pero al mismo tiempo según los estudios consultados se constatan diferencias de género, lo que hace pensar en una influencia de los roles de género y de cómo éstos van construyendo su identidad a partir de las relaciones sociales en los entornos digitales derivados de los procesos socioculturales.

En la misma forma, el modelo reveló un efecto directo positivo de las habilidades digitales de los jóvenes con el acceso y uso de Internet, por lo que se asume que los adolescentes durante su educación desarrollan y realizan ciertas competencias y/o habilidades digitales como parte de su formación. Por su parte, la Reforma Integral de Educación en México (RIEB), citado en la UNESCO (2011), explica que parte de las competencias que el estudiante debe adquirir durante su formación es la búsqueda, selección, análisis y evaluación proveniente de diversas fuentes; además de aprovechar los recursos tecnológicos a su alcance coma medios para comunicarse, obtener información y construir conocimiento. Sin embargo, el estudio empírico León, D., et al., (2014) evidenció una falta de dominio en este rubro cuando se analizó la búsqueda de información en diferentes medios digitales, sólo el $39 \%$ de los estudiantes dijo que siempre y casi siempre suele tener la habilidad de buscar en varios sitios para deberes escolares; mientras que el $33 \%$ consulta varios sitios, pero solo selecciona uno. Un $22 \%$ selecciona información en un solo sitio y un $35 \%$ solo busca en un sitio y copian la información tal cual.

Siguiendo en el informe de León, D., et. al., (2014) se mostró que los porcentajes más elevados que se presentaron en el eje de respuestas "algunas veces" y "nunca", donde alrededor del $25 \%$ y $27 \%$ de los jóvenes afirmaron tanto que "han echado solo un vistazo" como "explicar cómo se llevó a cabo la tarea" una cantidad de veces promedio al $26 \%$. En síntesis; el balance se encuentra distribuido en cuatro partes semejantes, la primera mitad se divide entre $26 \%$ no se esmera en su tarea contra el $20 \%$ que si lo hace. El $35 \%$ dice que si lee el documento contra $33 \%$ que no lo lee.

Lo anterior nos indica que existe un proceso de aceptación del cambio, entre aquellos alumnos que si utilizan las herramientas digitales para fines escolares y aquellos que lo están intentando. De igual forma, es posible enunciar que, si bien los jóvenes bajo estudio cuentan con la habilidad y dominio del uso de las TIC, es preciso hacer énfasis en un pensamiento crítico y reflexivo del adolescente de secundaria a fin de que pueda hacerse un uso más favorable de estas herramientas tecnológicas y el Internet.

De este modo, la incorporación de las TIC en la educación contribuye a que los estudiantes desarrollen la competencia digital que implica ser una persona autónoma, eficaz, responsable, crítica y reflexiva al seleccionar, tratar y utilizar la información y sus fuentes, además de sus herramientas tecnológicas (Area, 2009). Por su parte, Revuelta (2011) explica que "la competencia digital se sustenta en las competencias básicas en materia de TIC: el uso de ordenadores para obtener, evaluar, almacenar, producir, presentar e 
intercambiar información y comunicarse y participar en redes de colaboración a través de internet".

Paradójicamente, en otros estudios se menciona que algunas características de esta generación interactiva, es que se definen a sí mismos como autodidactas, con la capacidad de aprender y desaprender en el uso de los avances y recursos tecnológicos, (Prensky, 2001, 2004, 2006, y 2009; García, et. al. 2007, citados en León, D., et al., 2014). Por su parte, la aportación de Bringué; Sádaba y Rodríguez (2009) señala en términos generales que la generación interactiva Iberoamericana se define como autónoma por el uso mayoritariamente en solitario que hace de Internet, también se reconocen como autodidacta: la mitad de los internautas contesta de manera afirmativa la posibilidad "he aprendido solo a utilizar el Internet.

Estos resultados indican que el perfil de los estudiantes de secundarias de Hermosillo, México, se caracteriza primordialmente por los procesos de sociabilidad en línea que por una alfabetización digital. En otra interpretación, pese a que hubo una relación positiva de los factores de socialización en línea y habilidades digitales con el acceso y uso de Internet, podría asumirse que la socialización en línea para los jóvenes bajo estudio impera sobre una alfabetización digital encaminada a desarrollar habilidades digitales de manera crítica en un contexto educativo para el estudiante. En la misma línea, se afirma que los procesos de sociabilidad para los adolescentes son cruciales debido a las formas en la que hoy en día se han configurado los procesos de interacción en un entorno digital, o habría que decir, que es la posibilidad de la comunicación la que resulta central y esta queda garantizada con el acceso y uso de Internet. De acuerdo con Calvo (1996) se podría mantener que a mayor relación con las amistades disminuye el tiempo dedicado al estudio, lo que coincide con los resultados obtenidos del modelo estructural propuesto.

Es importante aclarar que una de las limitaciones que presenta el estudio es que la muestra la constituyen únicamente estudiantes de secundarias públicas mexicanas, por lo que se advierte que, sería conveniente, en futuros estudios, ampliar la muestra y extenderla a otros sectores de la población juvenil para contrastar o en su defecto, confirmar los resultados y lograr una generalización de los datos. Por otra parte, es preciso mencionar que el modelo propuesto hipotético es limitado en cuanto al número de factores que subyacen al mismo, por lo que sería de utilidad incluir el mayor número de factores posibles para lograr un modelo parsimonioso.

\section{REFERENCIAS}

Arnett, J.J. (1995). Broad and narrow socialization: The family in the context of a cultural theory, en: Journal of Marriage and the Family, 57: 617-628 
Area, Manuel. (2009). Introducción a la tecnología educativa. Islas Canarias, España: Universidad de La Laguna. Recuperado de https://campusvirtual.ull.es/ocw/file.php/4/ebookte.pdf

Ballesta, J., Lozano, J \& Cerezo, M. (2014). El uso y consumo de TIC en el alumnado autóctono y extranjero de Educación Secundaria Obligatoria de la Región de Murcia. Revista de Educación a Distancia, 41. Número especial "Interculturalidad en el nuevo paradigma educativo". Consultado el 10/11/2017 de: http://www.um.es/ead/red/41/ballesta.pdf

Ballesta, J. et al. (2015). Internet, redes sociales y adolescencia: un estudio en centros de educación secundaria de la región de Murcia. Revista Fuentes, 16, junio, pp. 109-130. [Fecha de consulta: 10/11/2017]. http://www.revistafuentes.es/

Bartolomé, J. Redes sociales y cuidado de la intimidad en adolescentes y familias: Una propuesta educativa. Murcia: Universidad de Murcia, 2014.

Bentler, P. M., \& Bonett, D. G. (1980). Significance tests and goodness of fit in the analysis of covariance structures. Psychological Bulletin, 88, 588-606

Bentler, P. M. (1990). Comparative fit indexes in structural models. Psychological Bulletin, 107, 238-246.

Bentler, P. M. (1992). On the fit of models to covariances and methodology to the Bulletin. Psychological Bulletin, 112, 400-404.

Bernete, F. (2010): 'Usos de las TIC, relaciones sociales y cambios en la socialización de las y los jóvenes, Revista de Estudios de Juventud, (88), 97-114. En línea: http://www.injuve.es/sites/default/files/RJ88-08.pdf

Bernal, C. \& Angulo, F. (2012). Interacciones de los jóvenes andaluces en las redes sociales. Comunicar, 20 (40), 25-30.

Bernal, A. \& Rivas, S., (2012). Un análisis de la realidad española a partir de la Encuesta. La familia, recurso de la sociedad, 2011. Investigación Internacional sobre la familia.

Berger, P; Luckman, T. (1968). La construcción social de la realidad. Buenos Aires: Amorrortu

Bringué, X \& Sádaba, C. (2009). La Generación interactiva en España. Niños y adolescentes ante las pantallas. Madrid: Ariel-Fundación Telefónica.

Bringué, X., Sádaba, C. y Tolsá, J. (2011). La generación interactiva en Iberoamérica 2010. Niños y adolescentes ante las pantallas. Madrid: Fundación Telefónica. 
Calvo, A. M. 1996 «Videojuegos: Del juego al medio didáctico», en Redes de comunicación, redes de aprendizaje, J. Salinas (coord), Edutec 95. Mallorca: Servicio de publicaciones de las Universidad de las Islas Baleares.

Camacho Yáñez, Iliana; Gómez Zermeño, Marcela Georgina; Pintor Chávez, María Manuela (2015). Competencias digitales en el estudiante adulto trabajador Revista Interamericana de Educación de Adultos, vol. 37, núm. 2, julio-diciembre, 2015, pp. 10-24 Centro de Cooperación Regional para la Educación de Adultos en América Latina y el Caribe Pátzcuaro, México.

Contreras-Cázarez, C.R., León, G.A., Moreno, D.E. (2014) Internet en jóvenes de secundaria. Un estudio de sociabilidad en las relaciones interactivas. Revista Internacional de Tecnologías en la Educación. Vol. 1, Número 1, 2014. Common Ground. ISSN: 2386-8392

Contreras, Cazarez, C. R., (2017) "Dependencia de Internet en el Uso de Dispositivos Móviles. Editorial Académica Española. ISBN 978-620-2-23443-6.

Colás, P., González, T. \& de Pablos, J. (2013). Juventud y redes sociales: motivaciones y usos preferentes. Comunicar, 20(40), 15-23.

Douglas, M., \& Isherwood, B. (1996); The World of Goods: Towards an Anthropology of Consumption; London, Routledge.

Fanni, F., Rega, I., Van Zyl, I., Cantoni, L. \& Tardini, S. (2010) Investigating Perception Changes in Teachers Attending ICT Curricula through Self-Efficacy. Proceedings ICTD 2010.

Fumero, A. y Espíritusanto, O. (2012). Jóvenes e infotecnologías. Entre nativ@ y digitales. Madrid: INJUVE.

Fundación Orange (2012). España. Informe anual 2012 sobre el desarrollo de la sociedad de la información en España. Madrid: Fundación Orange Extraído el 13 de septiembre de 2017 de http://www.proyectosfundacionorange.es/docs/ eE2012.pdf

Fundación Pfizer (2009). La juventud y las redes sociales en Internet. Madrid Fundación Pfizer. Extraído el 13 de septiembre de 2017 de http://www.asociacionplazadelcastillo.org/Textosweb/INFORME FINAL Encu esta Juventud y Redes Sociales.pdf 
Fundación Telefónica (2012). La Sociedad de la Información en España 2012. Madrid: Ariel. Extraído el 18 de septiembre de 2017 de: https://www.fundaciontelefonica.com/arte cultura/publicacioneslistado/pagina-item-publicaciones/?itempubli=176

García, F; Bringué, X., RIAL, (2007), Educar hij@s interactiv@s.

Gross, E. (2004). Adolescent Internet use. What we expected, what we report?. Applied Developmental Psychology, 25, 633-649.

Gutiérrez, A., y K. Tyner (2012), “Educación para los medios, alfabetización mediática y competencia digital", en Comunicar: Revista Científica Iberoamericana de Comunicación y Educación, núm. 38, pp. 31-39.

Grusec, Je.; Hastings, Pd. (eds.). (2007). Handbook of socialization. New York: Guilford.

Halpern, D.F. (2008). Is intelligence critical thinking? Why we need a new definition of intelligence. In P.C. Kyllonen, R.D. Roberts \& L. Stankov (Eds.), Extending intelligence. Enhancement and new constructs (pp. 157- 182). New York: Lawrence Erlbaum Associates

Hernández Fernández, C. y Alcoceba Hernando, J.A. (2015): Socialización virtual, multiculturalidad y riesgos de los adolescentes latinoamericanos en España, Icono 14, volumen (13), pp. 116-141. doi: 10.7195/ri14.v13i2.787

Hillaker, B. H., Brophy-Herb, H. E., Villarruel, F. A. y Haas, B. E. (2008): "The contribution of parenting to social competences and positive values in middle school youth: positive family communication, maintaining 27 standards, and supportive family relationships", Family Relations 57, pp. 591- 601.

Huertas, A. (2012). Procesos de sociabilidad e identidades en Internet: una aproximación a partir del estudio de contextos sociales multiculturales juveniles en España. Cogo, D., ElHajji, M. \& Huertas, A. (Coords.). Diásporas, migrações, tecnologias da comunicação e identidades transnacionais. Barcelona, InComUAB.

IAB México (Interactive Advertising Bureau), (2012). Es un organismo internacional que representa a la industria de la publicidad interactiva. Encargado de realizar estudios sobre Internet y medio publicitarios en México, consultado el 19 de septiembre de 2017 en: http://www.iabmexico.com/Medioteca/EstudiosMercado

León-Duarte, G; Caudillo, D.Y., Contreras-Cazarez, C.R. y Moreno, E. (2014). Internet seguro y jóvenes de secundaria en México. Hermosillo: Universidad de Sonora. 
León-Duarte, G; Caudillo R. D.; Contreras-Cázarez, C., Moreno C. D. (2015) Jóvenes y medios digitales móviles en México. Un estudio de variables asociadas en perspectiva Interdisciplinar. Primera edición. PEARSON EDUCACIÓN. México.

León-Duarte, G; Contreras Cázarez, CR; Moreno Carrillo, D. (2016): -Probando modelos interdisciplinares inclusivos en la dependencia de Internet en Jóvenes. Nuevas variables asociadas\|. Revista Latina de Comunicación Social, 71, pp. 616 a 631 http://www.revistalatinacs.org/071/paper/1112/32es.html

DOI:10.4185/RLSC-201

Lozano, A. (2010), Tecnología educativa y redes de aprendizaje de colaboración, México, Trillas.

Maccoby, EE. (2007). Historical overview of socialization research and theory. En: Grusec, Je; Hasting, Pd. (eds.). Handbook of socialization: Theory and research (pp.1341). New York: Guilford Press

Muñoz, L., \& Antón M., (2017) Estudio de uso y actitudes de consumo de contenidos digitales, consultado el 11 noviembre 2017 en: https://www.ontsi.red.es/ontsi/sites/ontsi/files/Uso\%20y\%20actitudes\%20de\% 20consumo\%20de\%20contenidos\%20digitales.\%20Julio\%202017.pdf

Organización para la Cooperación y el Desarrollo Económico (OCDE) (2010) Habilidades y competencias del siglo XXI para los aprendices del nuevo milenio en los países de la OCDE.

Organización para la Cooperación y el Desarrollo Económico (OCDE) (2011). PISA 2009 Results: Students On Line: Digital Technologies and Performance (Volume VI). OECD Publishing. Recuperado en noviembre del 2017 de: http://www.oecd.org/edu/school/programmeforinternationalstudentassess mentpisa/pisa2009resultsstudentsonlinedigitaltechnologiesandperformancev olumevi.htm

Prensky, M. (2001). Nativos e Inmigrantes Digitales. Madrid: Institución Educativa SEK. Recuperado de: $\quad$ https://www.marcprensky.com/writing/PrenskyNATIVOS\%20E\%20INMIGRANTES\%20DIGITALES\%20(SEK).pdf

Prensky, M. (2004). The emerging online live of the digital native: What they do differently because of technology, and how they do it. Sin publicar. Recuperado de: 
http://www.marcprensky.com/writing/Prensky-

The Emerging Online Life of the Digital Native-03.pdf

Prensky, M. (2006). Don't bother me mom, I'm learning! St. Paul: Paragon House.

Prensky, M. (2009). Let' s Be "Digital Multipliers" Eliminating the digital divide is something Educators can do. Educational Technology. Recuperado de: http://www.marcprensky.com/writing/Prensky-Lets Be Digital MultipliersET-01-09.pdf

Quintana, María; Cortada, Meritxell; Riera, Jordi. (2012). Internet navigation and information search strategies: How do children are influenced by their participation in an intensive ICT project. International Journal of Technology \& Design Education, vol. 22, núm. 4, pp. 513529. http://dx.doi.org/10.1007/s10798-011-9158-4

Revuelta, F. (2011), “Competencia digital: desarrollo de aprendizajes con mundos virtuales en la Escuela", en http://www.edutec.es/revista/index.php/edutece/article/view/397/133

Reyero, D., García Aretio, L., Hernández Serrano, M. J. y Ovide, E. (2011). Autonomía y responsabilidad en el contexto de la sociedad de las tecnologías de la información y la comunicación. Ponencia presentada en el XXX Seminario Interuniversitario de Teoría de la Educación. Barcelona.

Ruíz Corbella, M., Juana O., (2013) Redes sociales, identidad y adolescencia: nuevos retos educativos para la familia. Estudios Sobre Educación / vol. 25 / 2013 / 95-113. Consultado el 19 d Septiembre d 2017, en: https://www.unav.edu/publicaciones/revistas/index.php/estudios-sobreeducacion/article/viewFile/1883/1753

Sánchez Burón, A. y Álvaro Martín, A. (2011). Generación 2.0 Hábitos de uso de las redes sociales en los adolescentes de España y América Latina. Madrid: Universidd Camilo José Cela. Extraído el 19 de septiembre de 2017, de https://www.mecd.gob.es/dam/jcr:bd141f1f-e118-4a1a-a7c501706f3e8d55/generacion-ucjc.ppt \#256,1, Diapositiva 1

Shetzer, H. \& Warschauer, M. (2000). An electronic literacy approach to network-based language teaching. In M. Warschauer \& R. Kern (Eds.), Network-based language teaching: Concepts and practice (pp. 171-185). New York: Cambridge University Press. 
Steiger, J.H. (1990). Structural model evaluation and modification: an interval estimation approach. Multivariate Behavioral Research, 25, 173-180.

St. Martín, J. (2007). "Socialization". The politics and History of a Psychological concept, 1900-1970. Tesis de maestría por la Universidad de Wesleyan. Disponible en: [7 de enero, 2013]

SEP (2011). Habilidades Digitales para Todos. Secretaría de Educación Básica. Reforma Integral para la Educación Básica. México: Secretaría de Educación Pública. Recuperado de: http://www.sep.gob.mx/es/sep1/Programa Habilidades Digitales para Tod os\#.U9HBZ I5Mmk, citado em UNESCO, 2011.

Organización de las Naciones Unidas para la Educación, la Ciencia y la Cultura (UNESCO), (2011). Informe sobre la Reforma Integral de Educación Básica en México. consultado el 20 de septiembre de 2017. http://unesdoc.unesco.org/images/0022/002265/226543s.pdf

Organización de las Naciones Unidas para la Educación, la Ciencia y la Cultura, (UNESCO), (2017). Informe sobre la Comisión de la Banda Ancha de la ONU, 2014. Consultado el 17 de septiembre de 2017 en: http://es.unesco.org/news/medio-mundo-estar\%C3\%A1-|\%C3\%ADnea-2017

Van Zyl, I. \& Rega, I. (2011) ICT attitudes in educational practice: a qualitative perspective. In. Proceedings of the Red-Conference: Rethinking Education in the Knowledge Society. Ascona, Switzerland.

Winocur, R. (2009). Robinson Crusoe ya tiene celular. México DF, Mexico: Siglo XXI editores.

\section{Para referenciar este artículo:}

Contreras Cázarez, C. R., Campa Álvarez, R. (2017). Caracterización del perfil de los estudiantes de secundarias en el acceso y uso de internet a partir de las TIC. EDUTEC, Revista Electrónica de Tecnología Educativa, 61. Recuperado de: http://dx.doi.org/10.21556/edutec.2018.61 